\title{
Carnets
}

Revue électronique d'études françaises de l'APEF

Première Série - 1 Numéro Spécial | 2009

Cultures littéraires : nouvelles performances \& développement

\section{Michel Houellebecq, Amélie Nothomb et Jacques Chessex: Performances sous contexte médiatisé}

\section{Corina da Rocha Soares}

\section{(2) OpenEdition}

Journals

Édition électronique

URL : http://journals.openedition.org/carnets/3795

DOI : $10.4000 /$ carnets.3795

ISSN : 1646-7698

Éditeur

APEF

Édition imprimée

Date de publication : 1 juin 2009

Pagination : 207-220

Référence électronique

Corina da Rocha Soares, « Michel Houellebecq, Amélie Nothomb et Jacques Chessex: Performances sous contexte médiatisé », Carnets [En ligne], Première Série - 1 Numéro Spécial | 2009, mis en ligne le 16 juin 2018, consulté le 20 avril 2019. URL : http://journals.openedition.org/carnets/3795 ; DOI : $10.4000 /$ carnets. 3795 


\title{
MICHEL HOUELLEBECQ, AMÉLIE NOTHOMB ET JACQUES CHESSEX: PERFORMANCES SOUS CONTEXTE MÉDIATISÉ
}

\author{
CORINA DA ROCHA SOARES \\ Universidade de Aveiro; FCT \\ cgwenaelle@gmail.com
}

\section{Résumé}

Dans notre société contemporaine fortement médiatisé, un écrivain doit s'acquitter d'une nouvelle performance : la promotion de son œuvre créée sous les projecteurs des medias. Nous proposons, de ce fait, d'analyser les relations dissemblables que trois auteurs contemporains d'expression française, issus de champs distincts (le Français Michel Houellebecq, la Belge Amélie Nothomb et le Suisse romand Jacques Chessex), nouent avec les médias et les effets du phénomène de la médiatisation sur la création littéraire de ces trois écrivains : leur choix des thèmes, du style, leurs options modales et génériques.

\begin{abstract}
In our contemporary highly mediatised society, a writer must assume a new performance: the promotion of his creation produced under the media's projectors. We propose, therefore, to analyse the unlike relationships that three contemporaneous authors of French expression, issued from distinguish camps (the french Michel Houellebecq, the belgium Amélie Nothomb and the romand swiss Jacques Chessex), establish with the media and the effects of the mediatisation's phenomenon on the literary creation of these three writers: their choice of the themes, style, modal and gender options.
\end{abstract}

Mots-clés: Littérature francophone contemporaine (France, Belgique, Suisse), Effets de médiatisation, Michel Houellebecq, Amélie Nothomb, Jacques Chessex

Keywords: Contemporary francophone literature (France, Belgium, Switzerland), Mediatisation's effects, Michel Houellebeq, Amélie Nothomb, Jacques Chessex 
La société contemporaine est un contexte social fortement soumis aux règles des mass-media: elle est donc médiatique, d'où l'importance du spectacle et de l'image. La littérature, considérée comme acte social, semble souffrir, elle aussi, les forces du champ journalistique, sans oublier les effets des forces économistes. Comme le disait Hubert Nyssen, " Le succès d'un livre n'est plus, aujourd'hui, simple affaire de talent. II est aussi tributaire d'un couple inquiétant, celui que forment promotion et médiatisation. " (Nyssen, 2005: 120). Jérôme Meizoz parle, d'ailleurs, d'un nouvel état du champ littéraire contemporain : la médiatisation des auteurs (Meizoz, 2004).

Subséquemment, il est maintenant exigé à un écrivain de s'acquitter d'une nouvelle performance: la promotion de son œuvre créée, de manière à correspondre aux attentes d'un public " déterminé " par les règles des media, à travers la présence de l'écrivain dans différents espaces médiatiques. Jean-Paul Sartre s'en était déjà rendu compte, en affirmant : "Nous sommes beaucoup plus connus que nos livres ne sont lus. Nous touchons les gens, sans même le vouloir, par de nouveaux moyens. " (Sartre, 1948 : 293). Observation reprise plus tard par Guy Konopnicki, lorsqu'il observe que " [...] l'écrivain n'est plus reconnu pour son livre, mais pour ses passages à la télévision. [...] Un écrivain doit crever l'écran. [...] L'acte d'écrire n'existe pas. II faut un livre associé à une image présentable à la télévision. " (Konopnicki, 2004: 107 et 111). Ainsi, être écrivain aujourd'hui est aussi prendre une posture envers les media et, comme le conclue Jean-Louis Louette, "SainteBeuve a désormais l'âge des médias, qui donnent tort à Proust : le moi profond de l'écrivain s'abolirait au profit de son moi médiatique. "(Louette, $2003: 6$ ).

Il conviendrait, alors, de se pencher sur le rôle d'un écrivain dans la diffusion de son œuvre littéraire. Citant Julien Gracq,

\begin{abstract}
L'écrivain dispose aujourd'hui de mille manières de se manifester qui portent souvent infiniment plus loin que ses livres, [comme] [...]: grosseur des caractères dans les journaux, fréquence des photographies, manchettes des revues, 'présidiums' de congrès d'écrivains, [...] 'ventes' littéraires publiques, dont on diffuse les chiffres, apposition de noms au bas de manifestes, grandes orgues radiophoniques, séances de signatures [...]. (Gracq, $1961: 46-47$ ).
\end{abstract}

D'où le rôle du paratexte (couverture, quatrième de couverture, etc.) et de l'épitexte (extérieur au livre, comme la publicité) (cf. NYSSEN, 2005).

Les trois auteurs choisis (Michel Houellebecq, Amélie Nothomb et Jacques Chessex), contemporains mais issus de milieux géographiques distincts, sont des écrivains dont la légitimité littéraire est prouvée par plusieurs indices objectifs de 
consécration ${ }^{1}$. Présences assidues dans les media, nous pourrons vérifier, cependant, que la relation que les trois nouent avec ces derniers est dissemblable, tout comme leur performance de promoteurs de leur œuvre littéraire.

Observons l'exemple actuel de l'écrivain Michel Houellebecq, incorporé dans le champ littéraire de la France de la seconde moitié du XXème siècle. Les années 80 ont vu l'essor des media, puis l'effet Pivot et le monde de l'édition est, actuellement, sous contrôle de groupes déjà implantés dans l'audiovisuel. N'oublions pas la révolution que fut la popularisation de la littérature avec, par exemple, l'emploi généralisé du format livre de poche, le Club «France Loisirs », ou les magasins Fnac. Parallèlement, apparurent une multitude de revues littéraires, comme Les Nouvelles Littéraires, Le Monde des livres, Le Magazine littéraire, La Quinzaine littéraire, des émissions littéraires télévisées, comme Lecture pour tous, Ouvrez les Guillemets, Apostrophes, La rage de Lire, Bouillon de Culture, Campus. Mais plus important encore est le phénomène des apparitions d'écrivains dans la presse non littéraire, à la télévision et à la radio.

Michel Houellebecq, lui, est un écrivain polémique, choquant, explosif, dérangeant, maître de l'art de la provocation, un scandaleux qui aime l'insolence. François Busnel dit de lui dans L'Express qu' " II est vrai que Michel Houellebecq, omniprésent dans les médias, n'y va pas de main morte. [...] L'attention ne se porte plus sur le livre, elle se concentre sur le personnage. “ (Busnel, 2001). On accuse l'auteur d'avoir les idées de ses protagonistes, comme l'anti-islamisme, et la pornographie, et c'est une des raisons qui firent que Michel Houellebecq soit exclu de la revue Perpendiculaire, en $1998^{2}$.

Par ailleurs, sa figure médiatique est très forte, comme le souligne Jean-François Patricola : "Les médias [...] en ont fait l'incontournable objet du moment, le produit marketing par excellence qu'il faudra posséder. " (Patricola, 2005 : 10). En effet, plusieurs faits aident à la médiatisation de l'auteur: la sortie de ses oeuvres en pleine rentrée littéraire, accompagnée de couvertures médiatiques; interviews dans la presse (ex. : Le Monde, Libération); participation à des revues littéraires telles que Les Inrockuptibles, Lire, L'atelier du roman; présences à la télévision dans des émissions littéraires ou non, comme Bouillon de culture, Campus ou encore Nulle part ailleurs - dont il parvint à retarder la fin; adaptation au cinéma et à la télévision de ses romans ; site officiel de l'écrivain, forums multiples et existence d'une association d'amis et une d'ennemis de l'écrivain sur le web, etc.

\footnotetext{
${ }^{1}$ Ils appartiennent à des maisons d'édition prestigieuses, il existe des études critiques sur leur œuvre, ils sont mentionnés dans des manuels d'histoire littéraire, ils sont lauréats de plusieurs prix littéraires, leurs chiffres de vente sont haut-placés, ils sont traduits en plusieurs langues, etc. ...

2 Selon la conclusion de Jérôme Meizoz, la posture de Michel Houellebecq “ manifeste [...] un nouvel état du champ littéraire contemporain : toute une génération d'écrivains nés dans l'ère de la culture de masse [...] assument désormais pleinement la mise en scène publique de l'auteur à travers les fréquentes polémiques portant sur leur personne et leurs écrits. L'échange littéraire s'étant peu à peu calqué sur les exigences de la publicité et de l'image [...], ces mises en scène sont devenues partie intégrante d'une nouvelle manière d'envisager l'existence publique de la littérature. " (Meizoz, 2004 : 202-203)
} 
Ses interviews sont, fidèles au personnage, toujours sulfureuses, comme l'interview de Didier Sénécal dans Lire, le 6 septembre 2001, qui enclencha un procès judiciaire fortement médiatisé, causé par ses propos racistes et islamophobes. II se fâcha, à cette occasion, avec le chef de rédaction de la revue Pierre Assouline qu'il accusa de tronquer ses propos. La médiatisation de son procès dura de février à octobre 2002 : "Tous les journaux, toutes les radios et toutes les télévisions ont informé le public de l'existence d'un scandale Houellebecq. " (Konopnicki, 2004 : 62). Une des conséquences de cette médiatisation fut, d'ailleurs, son retrait de la sélection pour le Prix Goncourt, en 2001, avec son roman Plateforme. Après le procès, Michel Houellebecq coupa radicalement avec les media, il fuit Paris et partit s'installer en Espagne. II vit actuellement en Irlande.

Toutefois, ses relations avec les media ne se résument pas à ces épisodes. Déjà, dans les années 80 , il construisait des projets de documentaires audiovisuels avec JeanChristophe Debar. Plus tard, même lorsqu'il n'aura pas encore écrit une ligne de son roman La Possibilité d'une île, les media en feront une polémique anticipée. A la même époque, le 27 avril 2004, son passage de Flammarion chez Fayard fut fortement médiatisé, ce qui poussa Patricola à l'appeler le « Zidane du livre ».

Ajoutons à cela la construction de son image ou « construction posturale ${ }^{3}$, selon le terme de Meizoz (Meizoz, 2004 :80): discret, voir mutique, fumeur, mal dans sa peau, un aspect simplet, une allure de «minable » (Patricola, 2005 :144), donc une « anti-posture » d'écrivain académique.

Examinons, alors, les effets de la médiatisation de Michel Houellebecq sur sa création littéraire. Tout d'abord, les thèmes houellebecquiens sont des problèmes du monde contemporain, comme la haine, la solitude existentielle, la dénonciation de la misère affective de l'homme contemporain, tels ceux que nous pourrions lire dans un journal qui fait part de l'actualité : "On doit tout mettre dans un roman, y compris ce qui nous vient de l'actualité, de la vie de chaque jour pendant que l'on écrit. Je crois me souvenir avoir entendu Michel [Houellebecq] soutenir la même chose. ", souligne Dominique Noguez (Noguez, 2003 : 179).

Outre les thèmes houellebecquiens de controverse et polémiques comme le tourisme sexuel, la pédophilie, l'anti-islamisme, etc., les romans de Houellebecq regorgent de références aux media et au populisme : "son écriture est publicitaire, composée de maximes et de slogans.“ (Patricola, 2005: 222). II est vrai qu'il critique fréquemment les media dans ses romans, comme Elle, Le Nouvel Observateur, Madame Figaro ou des programmes télévisés dans Plateforme, par exemple. Mieux encore, nous pouvons trouver des réflexions sur le médiatisme d'un écrivain et ses stratégies promotionnelles, comme est le cas de

\footnotetext{
${ }^{3}$ Rappelons que, selon la définition de Jérôme Meizoz, la posture est " le travail de figuration publique qu'accomplit l'auteur en situation officielle. " (Meizoz, 2004: 201)
} 
Rester vivant ou l'auto-fiction qu'est La Possibilité d'une île, avec le personnage Daniel1, alter ego du Houellebecq médiatique.

Son style, fortement critiqué par Jean-François Patricola (Patricola, 2005: 237, par exemple), pourrait être relié au discours médiatique car les phrases courtes abondent. Tout comme les medias, son objectif est de révéler les problèmes de l'humanité, et ce d'une façon la plus neutre possible ; voilà pourquoi d'aucuns accusent Houellebecq de provoquer par le choix de ses mots et de ses thèmes, alors qu'il n'est peut-être que le reflet de la société contemporaine. De plus, comme le remarque Dominique Noguez, "Houellebecq estime que les questions de style sont relativement secondaires par rapport au fond. " (Noguez, 2003 : 100). Quant à la banalité de ses formulations, Pierre Jourde, lui, explique la platitude terne de son écriture en observant judicieusement que I'

On reproche aussi à Houellebecq de ne pas savoir écrire, de ne pas avoir de style. [...] La platitude de Houellebecq constitue son arme stylistique, et il sait en faire un usage efficace. [...] Une œuvre qui stigmatise l'illusion du désir d'originalité se doit de s'exprimer de manière terne. Houellebecq parle d'individus moyens, indifférenciés, dans un langage moyen. (Jourde, $2002: 233$ ).

Selon Patricola, Michel Houellebecq recourt souvent à l'épiphrase, interrompant son récit par un retour brutal à la forme discursive, semblable au langage journalistique (Patricola, 2005 :264). De plus, comme le remarque Dominique Noguez (Noguez, 2003), le métalangage est très important dans l'écriture houellebecquienne, comme l'italique, la ponctuation. Mieux encore, comme un journaliste, il utilise des phrases courtes et apostrophe souvent le lecteur.

Effectuons, alors, une lecture comparative du phénomène de la médiatisation et de l'influence que celle-ci peut exercer sur la création littéraire contemporaine, sous des contextes géographiquement distincts, comme la Belgique francophone, avec la coqueluche des lettres belges, Amélie Nothomb.

Le sud et le sud-est de la Belgique sont une région linguistique de près de $39 \%$ de francophones (Gorceix, $2000: 9$ ). Dans les années 40-60, le champ littéraire d'expression française de Belgique subit la monopolisation de la France et plusieurs écrivains se tournent vers Paris, capitale de l'édition, même si les années 70 assistèrent à un retour à la «belgitude ${ }^{4}$, avec, par exemple, la romancière Françoise Mallet-Joris.

Toutefois, un constat bien sombre est établi à la fin du XXème siècle. Robert FrickX affirme qu' " en Belgique, l'édition est quasi inexistante, la critique quasi muette en ce qui concerne nos auteurs, [...] l'enseignement de la littérature, basé essentiellement sur l'étude

\footnotetext{
${ }^{4}$ Notion créée, en 1976, par le sociologue Claude Javeau et Pierre Mertens
} 
des écrivains français, prépare très mal à la connaissance des nôtres. " (Frickx, In Gorceix, 1997: 30). Bilan appuyé par Marc Quaghebeur qui ajoute: “ Aujourd'hui encore, le corpus des auteurs belges de langue française ne fait pas partie du programme scolaire imposé dans le secondaire. II n'est toujours pas obligatoire, dans toutes les universités, pour les futurs professeurs de français du royaume... "(Quaghebeur, 1997: 68).

Selon Paul Aron, la nationalité belge implique que l'écrivain doit choisir les instances de consécration les plus efficaces: "La situation périphérique de nos écrivains influence directement toutes les instances qui peuvent assurer leur notoriété: le choix d'une maison d'édition, le relais de la critique, la possibilité d'obtenir des prix, l'accès aux grands médias, [...] sont autant de moments de leur carrière sur lesquels pèse l'origine d'une énonciation souvent vécue en termes d'illégitimité. " (Aron, 1997: 111). En effet, les instances de légitimation belges de langue française qui impliquent une reconnaissance internationale, sont minimes: l'Académie Royale (fondée en 1920), la Foire Internationale du Livre de Bruxelles (1ํédition du 21 au 30 mars 1969), quelques prix littéraires (le prix Rossel étant le plus important), peu de presse (ex: TXT), etc. Ainsi, les écrivains de la Belgique francophone se soumettent à la force centrifugeuse de Paris.

Amélie Nothomb ne fait pas exception et ses relations avec les forces médiatiques françaises sont soudées. Ses rituels sont donnés en spectacle, ses bizarreries publicisées : écrire tôt le matin, à $4 \mathrm{~h}$, après une tasse de thé fort, à jeun ; sa graphomanie ; l'écriture de tous ses manuscrits dans de vieux cahiers; l'entrepôt de ceux-ci dans le «frigo »; être publiée annuellement à la rentrée; ses chapeaux extravagants et son maquillage à la geisha; le fait qu'elle mange des fruits pourris ou chante le Nô, comme elle le démontra sur des plateaux de la télévision; etc. Amélie Nothomb cultive sa figure médiatique, sa posture, son image de "gotic-geisha" (Caine, 2003: 71) puisque "le public veut des livres, mais plus encore des auteurs, qui soient des personnages, des figures." (Boura, 2003 : 201). Elle donne, en complicité avec les media, une image de rock-star gothique.

De plus, elle joue complètement dans sa promotion de vedette avec ses séances de dédicaces, de courrier et de rencontres avec ses lecteurs ${ }^{5}$, de présences à la télé, à la radio, dans la presse. Elle signe plusieurs manifestes et a fait partie des «Chiennes de garde». Elle soigne particulièrement ses poses photographiques, surtout celles choisies pour la jaquette de ses romans. Nothomb varie ses apparitions médiatiques en spectacles infantiles (n'a-t-elle pas mangé des fruits pourris dans le plateau de Laurent Ruquier ${ }^{6}$ ?) et d'autres de grande maturité (comme elle le montra chez Bernard Pivot, face au membre de l'Académie Française de laquelle elle reçut le Grand Prix du Roman ou dans l'émission de «Bouillon de

\footnotetext{
${ }^{5}$ Même si Amélie Nothomb déclare " S'il y a une secte [de ses lecteurs], je n'y suis vraiment pour rien, et une chose est certaine, c'est que je n'en suis pas le gourou. " (apud in Amanieux, 2005 : 298)

${ }^{6}$ Cf. émission On a tout essayé du 19/09/2000, sur France 2 et émission Tout le monde en parle de Thierry Ardisson de mars 2000 sur France 2
} 
Culture » du 08/09/2000). Sa médiatisation s'accrut lorsqu'elle reçut, en novembre 1999, ce grand Prix de l'Académie française, avec son roman Stupeur et tremblements (Nothomb, 1999). Ses œuvres sont adaptées au théâtre, au cinéma et même à l'opéra, elle est primée de plusieurs prix littéraires et le web regorge de sites sur sa personne. Sa médiatisation est si forte qu'en Automne 2005, sa statue de cire fut inaugurée au musée Grévin.

Ainsi, son travail d'écrivain devient la «création accomplie », "celle d'un écrivain quand son livre est édité: aimer publiquement son texte, recevoir pour lui les compliments, les quolibets, l'indifférence. [...] L'aimer jusqu'au bout. “, comme le résume un des personnages de son roman Acide sulfurique (Nothomb, 2005: 69).

$\mathrm{Si}$ les options génériques d'Amélie Nothomb sont très hétéroclites (contes fantastiques, tragi-comédies, auto-fiction, etc.), les thèmes récurrents dans l'oeuvre d'Amélie Nothomb seraient l'amour et la mort, ou, comme l'explique Baptiste Liger, le " mariage entre Eros et Thanatos, thème déjà sous-jacent de toute son œuvre " (Liger, 2006 : 37). La perte dramatique de l'enfance, étape divine de l'existence, est un autre thème cher à cette écrivain belge qui perçoit l'adolescence comme le premier trépas de l'être humain, puisqu'il y perd son innocence, d'où la présence d'autres thèmes tels que la peur, l'angoisse du néant et du vide.

Nothomb traduit dans ses romans les marques psychologiques par une narration à la lisière du fantastique et du surnaturel, car le surgissement de la souffrance dans une vie humaine est, pour elle, de l'ordre du basculement soudain et irréversible dans une forme de folie. Ses romans peuvent se rattacher à l'une des principales tendances littéraires belges, celle du "réalisme magique" qui, d'après Roland Mortier, relève de "la transgression des bornes du réel. [...] On parlera alors de 'réalisme magique' ou de 'fantastique réel', qui se distingue de la création proprement fantastique". (Mortier, apud Joiret, 1999: 3). Cette tendance belge se caractérise par "l'irrationnel, une sensibilité tournée vers le mystère des êtres et des choses, une curiosité tendue vers ce qui se cache derrière le miroir où se reflète le quotidien." ( Ibidem).

Les romans nothombiens sont toujours des récits brefs dont la mise en page regorge d'espaces blancs, reflet, selon Daniel Garcia, du problème de l'anorexie souffert jadis par Amélie Nothomb. En effet, le critique souligne le fait que son roman Journal d'Hirondelle (Nothomb, 2006) a "seulement 136 pages, imprimées en gros caractères entourés de marges généreuses, comme si la production nothombienne semblait menacée d'anorexie galopante. " (Garcia, 2006 : 34).

Il est vrai aussi que nous pourrons trouver quelques indices des effets de la médiatisation de cette femme écrivain dans ses œuvres. A ce propos, Michel David constate que 
Ce n'est pas non plus un hasard si Amélie Nothomb est devenue un écrivain célèbre de cette modernité féroce qui s'avance désormais vers des formes de communautarisme et de ségrégation qui empirent et qu'elle écrit [...] sous les auspices de notre société de consommation dérégulée qui pousse chacun(e) vers son objet privé, son objet de jouissance particulier, vers un 'toujours plus', fût-il ludique ou télévisuel, objet de mode etc., mais rabattant l'objet du désir sous l'objet du besoin, de l'envie conditionnée, c'est-à-dire dorénavant le plus souvent médiatisée. (David, 2006 : 122-123).

L'exemple le plus paradigmatique est son roman Acide Sulfurique (Nothomb, 2005) qui est une satire de la télé-réalité. Son style, d'ailleurs, peut aussi être relié au discours médiatique: la prédominance du dialogue, des sarcasmes, des narratives courtes, en prose, des phrases succinctes, des paragraphes dotés parfois d'une seule phrase; l'importance de la parole, de la chose dite; l'ampleur donnée à l'image, à l'apparence physique; bref, des thèmes de suprématie utilisés par les media de notre société contemporaine, comme l'avait déjà remarqué Lénaïk le Garrec (Garrec, 2003 : 69).

Terminons donc notre lecture comparative avec une analyse portée sur la Suisse romande, avec le Prix Goncourt Jacques Chessex.

La Suisse romande (19\% de la population) est marquée par sa religion protestante et calviniste, qui oblige à l'introspection, au recueillement, à la discrétion et à l'effacement ${ }^{7}$. La composante religieuse est, en effet, un facteur déterminant. Le champ littéraire romand de la seconde moitié du XXème siècle est marqué par l'existence d'une presse et de maisons d'édition francophones qui souffrent la concurrence de la France, comme les éditions Bertil Gallan (1972-1982), dont les relations avec la presse furent soignées et où Jacques Chessex a collaboré ${ }^{8}$. Dès 1987, le Salon du Livre de Genève contribua à donner plus de visibilité aux écrivains suisses romands. Selon Roger Francillon (Francillon, 1998), le champ littéraire romand de la seconde moitié du XXème siècle se divise en trois étapes : 19681978 : refondation et affirmation de la «littérature romande »; 1978-1986 : apparition de plusieurs pôles éditoriaux; 1986-1996: spécialisation éditoriale, en voie de saturation et internationalisation de la littérature suisse romande. En outre, les années 78-86 sont la décennie de la «starisation", de la «popularisation» de la littérature romande, avec, notamment, des dossiers spéciaux dans des revues et des entretiens médiatiques, dont le plus célèbre est «Écrivains d'aujourd'hui » (Francillon, $1998: 73$ ).

Dans la seconde moitié du XXème siècle, la Suisse romande avait plusieurs émissions littéraires télévisées (ex. «Lecture à vue », «En toutes lettres » ou « Livre à

\footnotetext{
${ }^{7}$ Cf. Monnier, Jean-Pierre, In Gorceix, 1997: 157

${ }^{8}$ Galland publia aussi la revue annuelle Écriture avec Jacques Chessex, qui avait déjà été rédacteur principal de la revue Pays du Lac.
} 
vous ») et radiophoniques (ex : « La vie littéraire », " La semaine littéraire », etc.). Plusieurs œuvres romandes se voient aussi adaptées à la télévision.

La reconnaissance littéraire, en Suisse romande, est soumise à trois facteurs : les prix littéraires, la présence de l'auteur dans une anthologie et la mention de son nom dans des revues étrangères. II est vrai qu'il existe 1.4 fois plus de prix en Suisse romande qu'en France, dont le plus médiatique est le Prix Rambert. Cependant, seuls les écrivains publiant en France, comme Jacques Chessex, sont reconnus en France, situation déjà déplorée par Jean-Pierre Monnier :

L'audience de notre littérature en Suisse romande a toujours été bonne et, malgré les feuilletons télévisés, elle l'est encore. Mais l'intérêt des lecteurs est toujours allé de préférence aux écrivains français de France. D'ailleurs, nos universités (il y en a quatre de langue française) ignorent presque tout des littératures francophones hors de France, et, malgré quelques candidats au doctorat qui s'intéressent à nos auteurs, la littérature de Suisse romande elle-même y est à peine enseignée. (Monnier, In Gorceix, 1997: 159).

Jacques Chessex, cohérent avec sa situation géographique, est un écrivain introverti qui vit " à l'écart des agitations de la vie sociale." (Armel, avril $2007: 90)$ et qui tourne donc le dos à la médiatisation. L'auteur le confesse lui-même : " Je me méfie des évidences, de ce qui est donné sans discussions, de ce qui est trop vite lumière, des écrivains illustres: cela veut dire qu'ils sont trop clairs. " (Armel, avril 2007 : 93). Dans son recueil de trente trois petits textes intimes intitulé Le Désir de Dieu (Chessex, 2005), Jacques Chessex s'exprime à la première personne et démontre sa culture calviniste. Dans ces textes, il existe quelques passages qui prouvent que Chessex est discret, à l'ombre de sa popularité. II est même contre l'ordre du jour en ce qui concerne la mode actuelle selon laquelle l'écrivain doit être à la lumière des projecteurs et doit émigrer à Paris pour se faire connaître. En effet, il s'insurge contre la société et le champ éditorial qui l'entoure: " Dire non aux fâcheux qui insistent pour me convaincre de tel écrit; non à tel esprit du temps... " (Chessex, 2005: 213). Chessex compare l'écrivain à la chouette effraie: " C'est un oiseau discret, il vit dans l'ombre, il regarde dans l'ombre: leçon pour un écrivain. [...] L'effraie est une apparition, un cadeau mobile de la nuit. Elle n'éclaire pas. Elle a sa propre lumière [...]. L'effraie n'émigre pas, ne change pas de territoire. “ (Chessex, 2005: 292-295). D'ailleurs, dans un long entretien avec Geneviève Bridel en 2002 (Chessex, 2002), Chessex exprimait déjà son désir total de liberté et son refus d'obligations, de contraintes, rejoignant l'idée qu'il préfère se maintenir dans les coulisses, plutôt que de s'exposer aux projecteurs des media. Postulat que l'on retrouve 
dans la bouche du narrateur de L'Eternel sentit une odeur agréable: " Je préfère l'effacement au paraître. "(Chessex, $2004: 40)$.

II expose, d'ailleurs, les dessous de l'édition dans Monsieur (Chessex, 2001 :181185). Dans L'Ogre (Chessex, 1973), il critique le journal La tribune, mentionne des journaux, accusés d'être agents de propagation du scandale du personnage Grapp qui utilisa un fouet pour contrecarrer une manifestation d'étudiants et quand on lit, plus loin: "II ne fait pas bon être indépendant sous nos climats. Pas bon rester farouche et intraitable dans la ville." (Chessex, 1973 : 182), le narrateur, en se référant au rat écrabouillé par un trolleybus, n'estil pas Jacques Chessex qui se rebute contre le status quo du monde littéraire, se positionnant comme un écrivain qui se veut être hors-champ?

II enclencha, en outre, plusieurs tapages médiatiques, comme fut le cas de son pamphlet fougueux Avez-vous déjà giflé un rat ?, (Chessex, 1997), réponse aux altercations d'un de ses anciens élèves ${ }^{9}$. Néanmoins, ironie du sort, il reçoit le Prix Goncourt avec L'Ogre en 1973 ou plus récemment, en 2007, le prix Jean Giono pour l'ensemble de son oeuvre, et son roman Monsieur, par exemple, est adapté au cinéma en 1986...

Quant aux effets des media dans ses œuvres, nous pourrons vérifier qu'ils existent à peine, puisque Jacques Chessex veut les fuir. Son écriture est le reflet de plusieurs traits de l'écrivain suisse qui sont la relation à la nature et aux traditions authentiques de sa région (d'où la présence de régionalismes), l'âme inquiète, le coeur insatiable, la fascination de l'absolu ${ }^{10}$. On y retrouve aussi l'influence du protestantisme par la présence de la sobriété, de la pudeur, de la retenue, de la discrétion, de la solitude morale, des passions rentrées, de l'introspection et du repli de soi. Au milieu de ces thèmes, tels que le « côté inéluctablement tragique de la condition humaine » (Jaton, 2001: 9), la terreur, la violence, la méfiance, le malaise et la cruauté existentiels, le refuge dans la nature, la mort, le suicide, la culpabilité, le puritanisme et la pudeur de la Suisse, l'érotisme « lié à une forme de calvinisme abrupt 》 (Jaton, 2001: 69), Jacques Chessex nous laisse plusieurs passages dans son écriture contre la médiatisation. Mais plusieurs de ses histoires pourraient être comparés aux «faitsdivers » du champ journalistique (suicides, meurtres, scandales, tragédies causées par des folies psychiatriques, etc.).

Selon Anne-Marie Jaton son style est baroque et janséniste et Jacques Chessex est avant tout un poète, son lyrisme étant présent aussi bien dans la prose que dans ses vers. L'écrivain utilise souvent la répétition, l'antithèse et l'oxymoron - ce qui rejoindrait presque le

\footnotetext{
${ }^{9}$ Rappelons aussi la polémique engendrée lors de l'émission télévisée controverse «A la découverte de la littérature française " de Henri Guillemin, contre lequel Jacques Chessex écrit «Je voudrais rappeler à $M$. Guillemin ».

${ }^{10}$ Chessex est sensible à son appartenance à une "race et à une terre" et il veut, "à partir d'un lieu précis, exprimer l'univers tout entier, la nature, les bêtes, tous les signes du vivant des origines et, [...] l'amour des corps et la mort, les deux axes principaux qui s'entrecroisent sans cesse. " (Jaton, 2001 : 9).
} 
langage journalistique - afin de montrer avec réalisme les "failles de l'univers" (Jaton, 2001 : 69). Son option modale s'est tournée vers la nouvelle et le récit bref (comme la structure des faits-divers dont nous avons déjà parlé) et le squelette de ses romans se répète, tout comme chez Amélie Nothomb :

Un personnage central est le seul maître de la narration [...]; insatisfait de ce qu'il est, il est saisi en ouverture de roman dans un moment de 'crise ', il est en quête d'une forme quelconque de transformation, de renaissance ou, plus tard, selon un terme que Chessex affectionne, de 'désencombrement'. II possède l'intime conviction que, pour aboutir, cette quête doit passer par le corps des femmes, même lorsque son objet, dans les romans plus récents en particulier, est la recherche d'une sorte de sainteté laïque. (Jaton, $2001: 81$ ).

Après toutes ces observations, nous pouvons donc conclure que Michel Houellebecq, Amélie Nothomb et Jacques Chessex, écrivains contemporains issus de contextes géographiques distincts, sont, toutefois, ancrés dans une époque où la médiatisation apporte ses contraintes sur le champ littéraire.

Toutefois, nous avons pu vérifier que la relation que les trois nouent avec les media est dissemblable, tout comme leur performance de promoteurs de leur œuvre littéraire. Houellebecq joue sur la polémique corrosive, la posture vitriolée ${ }^{11}$; Nothomb utilise à son profit les media pour la promotion de sa figure et de ses romans et Chessex préfère l'image d'un auteur qui s'éloigne des lumières médiatiques.

Ainsi, ces trois performances sous contexte médiatisé nous font penser à la classification des intellectuels élaborée, en 1987, par Eduard Brasey (Brasey, 1987 : 179180), qui utilisent deux stratégies médiatiques envers l'émission d'Apostrophes de Bernard Pivot, à savoir, paraître avec ferveur, dont l'exemple paradigmatique est Bernard Henri-Lévy et ne pas y apparaître, mais avec fracas, comme le fit Régis Debray : " il existe ceux qui vont, qui courent ou qui refusent de se rendre à 'Apostrophes' “:

1. l'absent (sic): n' apparaît pas dans les médias ;

2. le râleur ( $\operatorname{sic}$ ): apparaît pour les critiquer ;

3. le cathodique ( $\mathrm{sic}$ ) : se sert des médias avec modération, intelligemment ;

4. le clown (sic) : abuse des médias.

\footnotetext{
${ }^{11}$ Jérôme Meizoz inclue Houellebecq dans le groupe des auteurs qui "surjouent la médiatisation de leur personne et l'incluent à l'espace de l'œuvre: leurs écrits et la posture qui les fait connaître se donnent solidairement comme une seule performance" (Meizoz, 2004: 203).
} 
Dès lors, Michel Houellebecq nous semble tenir le rôle du clown râleur, Amélie Nothomb celui du clown cathodique et Jacques Chessex de l'absent.

De plus, nous avons trouvé plusieurs indices qui nous permettent d'affirmer que le contexte médiatisé provoque et incite à de nouvelles performances chez les écrivains contemporains et agit sur leur création littéraire, ce qui nous amène à réfléchir sur la définition d'Hubert Nyssen d'un auteur à succès, aujourd'hui assimilé à une star:

Pour un écrivain, vivre de sa plume [...], c'est toujours [...] la preuve d'une réussite. $\mathrm{Si}$, de surcroît, il accumule les best-sellers, s'il se prête à la curiosité de photographes [...], s'il fréquente [...] les lieux à la mode [...], s'il écrit dans des revues [...], s'il est de toutes les grandes messes audiovisuelles, si sa voix porte sur les ondes, alors il incarne le type même de l'écrivain arrivé. (Nyssen, 2005: 134) 


\section{Bibliographie}

AmanieuX, Laureline (2005). Amélie Nothomb, l'éternelle affamée. Paris : Albin Michel

ARON, PAUL (1997), "Les revues littéraires, média privilégié de l'identité culturelle ?". In : GORCEIX, Paul (1997). L'identité culturelle de la Belgique et de la Suisse francophones, actes du colloque. Paris : Honoré Champion, pp.109-120

BAINBRIgGe, Susan et Toonder, Jeanette den (ed.) (2003). Amélie Nothomb: Autorship, Identity and Narrative Practice. Peter Lang : New York

BOURA, Olivier (2003). Un siècle de Goncourt. s.I. : Arléa

BRASEY, Eduard (1978). L'effet Pivot. Ramsay : Paris

CAINE, Philippa (2003), "'Entre-deux' Inscription of Female Corporeality in the Writing of Amélie Nothomb". In BAINBRIGge, Susan et TOONDER, Jeanette den (ed.) (2003). Amélie Nothomb: Autorship, Identity and Narrative Practice. Peter Lang : New York, pp. 71-84

CHESSEX, Jacques (1973). L'Ogre. Lausanne : Editions Grasset \& Fasquelle, col. Les Cahiers Rouges

CHESSEX, Jacques (1997). Avez-vous déjà giflé un rat ?, un pamphlet. Yvonand: Bernard Campiche Editeur

CHESSEX, Jacques (2001). Monsieur. Paris : Grasset

CHESSEX, Jacques (2002). Transcendance et transgression, entretiens avec Geneviève Bridel. s.I. : La Bibliothèque des Arts, col. Paroles vives

CHESSEX, Jacques (2004). L'Eternel sentit une odeur agréable. Paris: Grasset et Fasquelle

ChesseX, Jacques (2005). Le Désir de Dieu. Paris : Grasset

DAVID, Michel (2006). Amélie Nothomb, le symptôme graphomane. Paris : L' Harmattan

FRANCILLON, Roger, (dir) (1998). Histoire de la Littérature en Suisse romande, vol.4 "La littérature romande aujourd'hui“. Lausanne : Editions Payot Lausanne

GARREC, Lénaïk le (2003), "Beastly Beauties and Beautiful Beasts“. In BAINBRIGGE, Susan et TOONDER, Jeanette den (ed.) (2003). Amélie Nothomb: Autorship, Identity and Narrative Practice. Peter Lang : New York, pp. 63-70

GoRCEIX, Paul (1997). L'identité culturelle de la Belgique et de la Suisse francophones, actes du colloque. Paris : Honoré Champion

GORCEIX, Paul (2000). Littérature francophone de Belgique et de Suisse. Paris : Ellipses

GRACQ, Julien (1961). "La littérature à l'estomac" in Préférences. Paris : José Corti

JATON, Anne-Marie (2001). Jacques Chessex, La Lumière de L'Obscur. Genève : éditions Zoé

JOIRET, Michel et BERNARD, Marie-Ange (1999). Littérature belge de langue française. Bruxelles : Ed. Didier Hatier

JoURDE, Pierre (2002). La littérature sans estomac. Paris : L'esprit des Péninsules

KONOPNICKI, Guy (2004). Prix littéraires : la grande magouille. Paris : Jean-Claude Gawsewitch Editeur

LOUETTE, Jean-François (2003), "Présentation“. In LOUETTE, J. François et RochE, RogerYves (2003). Portraits de l'écrivain contemporain. Seissel : Editions Champ Vallon

MEIZOZ, Jérôme (2004). L'oeil sociologique et la littérature, essai. Genève : Slatkine Erudition NOGUEZ, Dominique (2003). Houellebecq, en fait. Paris : Fayard 
NotнOMB, Amélie (1999). Stupeur et tremblements. Paris : Albin Michel

NOTHOMB (2005). Acide sulfurique. Paris : Albin Michel

NothOMB (2006). Journal d'Hirondelle. Paris : Albin Michel

NYSSEN, Hubert (2005). Du texte au livre, les avatars du sens. Paris: Armand Collin

PATRICOLA, Jean-François (2005). Michel Houellebecq ou la provocation permanente. Paris: Ecriture

QUAGHEBEUR, Marc (1997), "L'identité ne se réduit pas à la langue“. In : GoRCEIX, Paul (1997). L'identité culturelle de la Belgique et de la Suisse francophones, actes du colloque. Paris : Honoré Champion, pp.59-105

SARTRE, Jean-Paul (1948). Qu'est-ce que la littérature ?. Paris: Gallimard

\section{Articles de presse}

ARMEL, Aliette. "Jacques Chessex, autoportrait d'un hérétique ". In : Le Magazine Littéraire, n-463, avril 2007, pp.90-95

BuSNEL, François. "Le fabuleux destin de Michel H. “. In : L'Express, 30/08/2001

GARCIA, Daniel. "Les silences d'Amélie“. In : Lire, no348, septembre 2006, pp.32-38

LIGER, Baptiste."Le Nothomb nouveau est arrivé“. In : Lire, no348, septembre 2006, p.37

VIRY, Marin de. "Houellebecq, portrait de l'artiste en gnome nécrophile". In : Revue des deux Mondes, no 12 du 01/12/2005 